\title{
On Nonperturbative Techniques for Thermal Radiation Effect on Natural Convection past a Vertical Plate Embedded in a Saturated Porous Medium
}

\author{
O. D. Makinde ${ }^{1}$ and R. J. Moitsheki ${ }^{2}$ \\ ${ }^{1}$ Faculty of Engineering, Cape Peninsula University of Technology, P.O. Box 1906, \\ Bellville 7535, South Africa \\ 2 School of Computational and Applied Mathematics, University of the Witwatersrand, \\ Private Bag 3, Wits 2050, South Africa \\ Correspondence should be addressed to O. D. Makinde, makinded@cput.ac.za
}

Received 16 March 2008; Revised 30 May 2008; Accepted 24 June 2008

Recommended by Carlo Cattani

In this article, the heat transfer characteristics of natural convection about a vertical permeable flat surface embedded in a saturated porous medium are studied by taking into account the thermal radiation effect. The plate is assumed to have a power-law temperature distribution. Similarity variables are employed in order to transform the governing partial differential equations into a nonlinear ordinary differential equation. Both Adomian decomposition method (ADM) and He's variational iteration method (VIM) coupled with Padé approximation technique are implemented to solve the reduced system. Comparisons with previously published works are performed, and excellent agreement between the results is obtained.

Copyright (C) 2008 O. D. Makinde and R. J. Moitsheki. This is an open access article distributed under the Creative Commons Attribution License, which permits unrestricted use, distribution, and reproduction in any medium, provided the original work is properly cited.

\section{Introduction}

Heat transfer from different geometrics embedded in porous media has many engineering and geophysical applications such as geothermal reservoirs, drying of porous solids, thermal insulation, enhanced oil recovery, packed-bed catalytic reactors, cooling of nuclear reactors, and underground energy transport [1]. Nakayama and Koyama [2] studied free convection over a vertical flat plate embedded in a thermally stratified porous medium by exploiting the similarity transformation procedure. Cheng and Minkowycz [3] studied the steady free convection about a vertical plate embedded in a porous media using the boundary layer assumptions and Darcy model by the similarity method. Cheng [4] extended the work by studying the effect of lateral mass flux with prescribed temperature and velocity as power law on the vertical surface. Other investigators [5-8] studied some similar porous medium 
cases using Darcy and Boussinesq approximations with different power-law velocity and temperature variations at the boundaries.

Meanwhile, the boundary layer equations for free-convective flow through a porous medium constitute a nonlinear problem. The theory of nonlinear differential equations is quite elaborate and their solutions are of practical relevance in the engineering sciences. Several numerical approaches have been developed in the last few decades (e.g., finite differences, spectral method, shooting method, etc.) to tackle this problem. More recently, the ideas of classical analytical methods have experienced a revival in connection with the proposition of novel hybrid numerical-analytical schemes for nonlinear differential equations. Among such trends are Adomian decomposition method (ADM) [9-12] and He's variational iteration method (VIM) [13-16] coupled with Padé approximation method [17] especially when dealing with boundary value problems [18]. These techniques, over the last few years, have proved themselves as a powerful tool and a potential alternative to traditional numerical techniques in various applications in science and engineering. This seminumerical approach is also extremely useful in the validation of purely numerical schemes.

The aim of the present work is to construct a nonperturbative solution for natural convection boundary layer flow through a porous medium on an unbound domain in the presence of radiation using both ADM and VIM coupled with Padé approximation technique. The chief merit of the methods is that they are capable of greatly reducing the size of computation work while still maintaining accuracy of the numerical solution. However, VIM gives successive approximations of high accuracy of the solution and VIM does not require specific treatments as in ADM for nonlinear terms. Both numerical and graphical results are presented and discussed quantitatively with respect to various parameters embedded in the problem.

\section{Mathematical formulation}

We consider the steady two-dimensional flow of an incompressible viscous fluid induced by a heated vertical plate embedded in a homogeneous porous medium of uniform ambient temperature $T_{\infty}$. The fluid is assumed to be Newtonian, and a constant fluid suction or blowing is imposed at the plate surface. Under Darcy and Boussinesq approximations, the governing boundary layer equations for this problem can be written as $[5,6]$

$$
\begin{gathered}
\frac{\partial u}{\partial x}+\frac{\partial v}{\partial y}=0 \\
\frac{\partial u}{\partial y}=\frac{g K \beta}{v} \frac{\partial T}{\partial y} \\
\rho c_{p}\left(u \frac{\partial T}{\partial x}+v \frac{\partial T}{\partial y}\right)=k \frac{\partial^{2} T}{\partial y^{2}}-\frac{\partial q_{r}}{\partial y} .
\end{gathered}
$$

Here, $u, v$ are the velocity components along $x, y$ coordinates, $\rho$ the fluid density, $T$ the temperature of the fluid, $c_{p}$ specific heat at constant pressure, $k$ the thermal conductivity, $v$ kinematic viscosity, $g$ the gravitational acceleration, $K$ permeability of the porous medium, and $\beta$ thermal expansion coefficient. It is also assumed that the temperature distribution of the plate is governed by the power law $T_{w}(x)=T_{\infty}+A x^{\lambda}$, where $A$ is a constant $>0$ for heated 
plate. Using Roseland approximation $[7,19]$, we takethe radiative heat flux as

$$
q_{r}=-\frac{4 \sigma}{3 \gamma} \frac{\partial T^{4}}{\partial y}
$$

where $\sigma$ is the Stefan-Boltzmann constant and $\gamma$ the mean absorption coefficient. Assume that the temperature differences within the flow are sufficiently small such that $T^{4}$ may expressed as a linear function of temperature

$$
T^{4} \approx 4 T_{\infty}^{3} T-3 T_{\infty}^{4}
$$

The boundary conditions are given by

$$
\begin{aligned}
T(x, 0) & =T_{w}(x), \quad v(x, 0)=V(x), \\
T(x, \infty) & =T_{\infty}, \quad u(x, \infty)=0 .
\end{aligned}
$$

We introduced the following similarity variables and parameters $[5,8]$ :

$$
\begin{gathered}
\Psi=\alpha R a_{x} F(\eta), \quad R a_{x}=\frac{g K \beta\left(T_{w}-T_{\infty}\right) x}{v \alpha}, T=T_{\infty}+A x^{\lambda} \theta(\eta), \\
N=\frac{16 \sigma T_{\infty}^{3}}{3 \gamma k}, \quad \eta=\left(\frac{y}{x}\right) R a_{x}^{1 / 2}, \quad \alpha=\frac{k}{\rho c_{p}}, \quad \theta=\frac{T-T_{\infty}}{T_{w}-T_{\infty}},
\end{gathered}
$$

where $R a_{x}$ is the modified local Rayleigh number. The continuity equation (2.1) is satisfied by the stream function $\Psi(x, y)$ defined by

$$
u=\frac{\partial \Psi}{\partial y}=\left(\frac{\alpha}{x}\right) R a_{x} F^{\prime}(\eta), \quad v=-\frac{\partial \Psi}{\partial x}=-\left(\frac{\alpha}{2 x}\right) R a_{x}^{1 / 2}\left[(\lambda+1) F+(\lambda-1) F^{\prime}\right]
$$

and (2.2) and (2.3) become

$$
F^{\prime \prime}=\theta^{\prime}, \quad \theta^{\prime \prime}+\frac{\lambda+1}{2(N+1)} F \theta^{\prime}-\frac{\lambda}{N+1} F^{\prime} \theta=0,
$$

where the primes denote differentiation with respect to $\eta, N$ is the Radiation parameter, and $\lambda$ is the temperature exponent. In view of (2.7), the boundary conditions (2.6) transform into

$$
\theta(0)=1, \quad \theta(\infty)=0, \quad F(0)=m, \quad F^{\prime}(\infty)=0 .
$$

The suction or injection speed at the plate surface becomes

$$
v(x, 0)=-\left(\frac{\alpha}{2 x}\right) R a_{x}^{1 / 2}(\lambda+1) F(0),
$$


where $m=F(0)$ is the suction or injection parameter according to $m>0$ or $m<0$, respectively. The entrainment velocity of the fluid is given by

$$
v(x, \infty)=-\left(\frac{\alpha}{2 x}\right) R a_{x}^{1 / 2}(\lambda+1) F(\infty) .
$$

Equation (2.9) together with the boundary conditions (2.10) can be easily reduced to give

$$
F^{\prime \prime \prime}+\frac{\lambda+1}{2(N+1)} F^{\prime \prime} F-\frac{\lambda}{N+1} F^{\prime 2}=0
$$

with

$$
F^{\prime}(0)=1, \quad F(0)=m, \quad F^{\prime}(\infty)=0,
$$

since it is very obvious from (2.9) and (2.10) that $F^{\prime}=\theta$ (i.e., the vertical velocity and the temperature profiles are identical). The local surface heat flux can be expressed as a function of the local Rayleigh and Nusselt numbers as

$$
N u_{x} R a_{x}^{-1 / 2}=-\theta^{\prime}(0)
$$

\section{Adomian decomposition method}

In order to explicitly construct approximate nonperturbative solutions of the problem described by (2.13) and (2.14), Adomian decomposition method well addressed in [9-11] is employed and implemented in Maple (a symbolic algebra package). We rewrite (2.13) in the form

$$
L_{\eta} F=\frac{\lambda}{(N+1)}\left(F_{\eta}\right)^{2}-\frac{(\lambda+1)}{2(N+1)} F F_{\eta \eta}
$$

where the subscript $\eta$ represents differentiation with respect to $\eta$ and the differential operator employs the first three derivatives in the form $L_{\eta}=d^{3} / d \eta^{3}$. The inverse operator $L_{\eta}^{-1}$ is considered a threefold integral operator defined by

$$
L_{\eta}^{-1}=\int_{0}^{\eta} \int_{0}^{\eta} \int_{0}^{\eta}(\cdot) d \eta d \eta d \eta
$$

Applying $L_{\eta}^{-1}$ to both sides of (3.1), using the boundary conditions in (2.14), we obtain

$$
F(\eta)=m+\eta+b \frac{\eta^{2}}{2}+a_{1} L_{\eta}^{-1}\left(F_{\eta}^{2}\right)-a_{2} L_{\eta}^{-1}\left(F F_{\eta \eta}\right)
$$


where $a_{1}=\lambda /(N+1), a_{2}=(\lambda+1) / 2(N+1)$, and $b=F^{\prime \prime}(0)$ is to be determined from the boundary condition at infinity in (2.14). As usual in Adomian decomposition method, the solution of (3.3) is approximated as an infinite series

$$
F(\eta)=\sum_{j=0}^{\infty} F_{j}
$$

and the nonlinear terms are decomposed as

$$
F_{\eta}^{2}=\sum_{j=0}^{\infty} H_{j}, \quad F F_{\eta \eta}=\sum_{j=0}^{\infty} G_{j}
$$

where $H_{j}, G_{j}$, are polynomials (called Adomian polynomials) given by

$$
\begin{aligned}
& H_{j}=\frac{1}{j !} \frac{d^{j}}{d S^{j}}\left[\left(\sum_{i=0}^{\infty} F_{\eta i} S^{i}\right)^{2}\right]_{S=0}, \\
& G_{j}=\frac{1}{j !} \frac{d^{j}}{d S^{j}}\left[\left(\sum_{i=0}^{\infty} F_{i} S^{i}\right)\left(\sum_{i=0}^{\infty} F_{\eta \eta i} S^{i}\right)\right]_{S=0} .
\end{aligned}
$$

Thus, we can identify

$$
\begin{aligned}
F_{0} & =m+\eta, \quad F_{1}=\frac{b \eta^{2}}{2}+a_{1} L_{\eta}^{-1}\left(H_{0}\right)-a_{2} L_{\eta}^{-1}\left(G_{0}\right), \\
F_{j+1} & =a_{1} L_{\eta}^{-1}\left(H_{j}\right)-a_{2} L_{\eta}^{-1}\left(G_{j}\right), \quad \text { for } j \geq 1 .
\end{aligned}
$$

Using Maple, we obtained a few terms approximation to the solution as

$$
\begin{aligned}
F_{1}= & \frac{1}{2} b \eta^{2}+\frac{(1 / 6) \lambda \eta^{3}}{N+1} \\
F_{2}= & \left(\frac{1}{60} \frac{\lambda^{2}}{(N+1)^{2}}-\frac{1}{60} \frac{(1+\lambda) \lambda}{(2 N+2)(N+1)}\right) \eta^{5} \\
& +\left(\frac{1}{12} \frac{\lambda b}{N+1}-\frac{(1+\lambda)((1 / 24)(m \lambda /(N+1))+(1 / 24) b)}{2 N+2}\right) \eta^{4}-\frac{1}{6} \frac{(1+\lambda) m b \eta^{3}}{2 N+2}
\end{aligned}
$$


and so on. Substituting (3.7) into (3.4), we obtain

$$
\begin{aligned}
F(\eta)= & m+\eta+\frac{1}{2} b \eta^{2}-\frac{1}{12} \frac{(b m+b m \lambda-2 \lambda) \eta^{3}}{N+1} \\
& +\frac{1}{96} \frac{1}{(N+1)^{2}}\left(\left(-2 b N-2 b+6 b \lambda N+6 b \lambda+b m^{2}+2 b m^{2} \lambda-2 m \lambda+b m^{2} \lambda^{2}-2 m \lambda^{2}\right) \eta^{4}\right) \\
& +\frac{1}{480} \frac{1}{(N+1)^{2}}\left(\left(-2 b^{2} N-2 b^{2}+6 b^{2} \lambda N+6 b^{2} \lambda-2 b m \lambda-5 b m \lambda^{2}+4 \lambda^{2}+3 b m-4 \lambda\right) \eta^{5}\right) \\
& +\mathrm{O}\left(\eta^{6}\right),
\end{aligned}
$$

where other terms up to $\mathrm{O}\left(\eta^{13}\right)$ were derived. Let $W_{L}=\sum_{j=0}^{L} F_{j}$ represent the decomposition series partial sum obtained, then $F(\eta)=\lim _{L \rightarrow \infty}\left(W_{l}\right)$.

\section{He's variational iteration method}

In 1978, Inokuti et al. [20] proposed a general Lagrange multiplier method to solve nonlinear problems, which was first proposed to solve problems in quantum mechanics. The modified method, or variational iteration method (VIM) proposed by He [13-16], has been shown to solve effectively, easily, and accurately a large class of nonlinear problems with approximations converging rapidly to accurate solutions. To illustrate the basic idea of the method, we consider the general nonlinear system

$$
L[F(\eta)]+N[F(\eta)]=g(\eta)
$$

where $L$ is a linear operator, $N$ is a nonlinear operator, and $g(\eta)$ is a given continuous function. The basic character of the method is to construct a correction functional for the system, which reads

$$
F_{n+1}(\eta)=F_{n}(\eta)+\int_{0}^{\eta} B(s)\left[L F_{n}(s)+N \tilde{F}_{n}(s)-g(s)\right] d s
$$

where $B$ is a Lagrange multiplier which can be identified optimally via variational theory, $F_{n}$ is the $n$th approximate solution, and $\widetilde{F}_{n}$ denotes a restricted variation (i.e., $\delta \widetilde{F}_{n}=0$ ). This technique provides a sequence of functions which converges to the exact solution of the problem. The initial values $F(0), F^{\prime}(0)$, and $F^{\prime \prime}(0)$ are usually used for selecting the zeroth approximation $F_{0}$. Consequently, the exact solution may be obtained by using $F(\eta)=$ $\lim _{n \rightarrow \infty} F_{n}$.

In what follows, we will apply the VIM for the problem in (2.13) to illustrate the strength of the method. The correction functional for (2.13) reads

$$
F_{n+1}(\eta)=F_{n}(\eta)+\int_{0}^{\eta} B(s)\left[F_{n}^{\prime \prime \prime}+\frac{\lambda+1}{2(N+1)} \widetilde{F}_{n}^{\prime \prime} \widetilde{F}_{n}-\frac{\lambda}{N+1} \widetilde{F}_{n}^{\prime 2}\right] d s .
$$


Making the above correction functional stationary with respect to $F_{n}$ yields the stationary conditions (Euler equations)

$$
\frac{\partial^{3} B}{\partial s^{3}}=0, \quad 1+\left.\frac{\partial^{2} B}{\partial s^{2}}\right|_{s=\eta}=0,\left.\quad \frac{\partial B}{\partial s}\right|_{s=\eta}=0,\left.\quad B(s)\right|_{s=\eta}=0 .
$$

Solving the above equations results in $B=-(1 / 2)(s-\eta)^{2}$, and (4.3) then becomes

$$
F_{n+1}(\eta)=F_{n}(\eta)-\frac{1}{2} \int_{0}^{\eta}(s-\eta)^{2}\left[F_{n}^{\prime \prime \prime}(s)+\frac{\lambda+1}{2(N+1)} F_{n}^{\prime \prime}(s) F_{n}(s)-\frac{\lambda}{N+1} F_{n}^{\prime 2}(s)\right] d s .
$$

We select the initial value $F_{0}(\eta)=m+\eta+(1 / 2) b \eta^{2}$ by using the conditions in (2.14), where $b=F^{\prime \prime}(0)$ is to be determined from the boundary condition at infinity in (2.14). Using (4.5), we obtain the next successive approximation as

$$
\begin{aligned}
F_{1}(\eta)= & \left(-\frac{1}{120} \frac{(1+\lambda) b^{2}}{2 N+2}+\frac{1}{60} \frac{\lambda b^{2}}{N+1}\right) \eta^{5}+\left(-\frac{1}{24} \frac{(1+\lambda) b}{2 N+2}+\frac{1}{12} \frac{\lambda b}{N+1}\right) \eta^{4} \\
& +\left(-\frac{1}{6} \frac{(1+\lambda) b m}{2 N+2}+\frac{1}{6} \frac{\lambda}{N+1}\right) \eta^{3}+\frac{1}{2} b \eta^{2}+\eta+m
\end{aligned}
$$

and after few iterations, we obtain

$$
\begin{aligned}
F(\eta)= & m+\eta+\frac{1}{2} b \eta^{2}-\frac{1}{12} \frac{(b m+b m \lambda-2 \lambda) \eta^{3}}{N+1} \\
& +\frac{1}{96} \frac{1}{(N+1)^{2}}\left(\left(-2 b N-2 b+6 b \lambda N+6 b \lambda+b m^{2}+2 b m^{2} \lambda-2 m \lambda+b m^{2} \lambda^{2}-2 m \lambda^{2}\right) \eta^{4}\right) \\
& +\frac{1}{480} \frac{1}{(N+1)^{2}}\left(\left(-2 b^{2} N-2 b^{2}+6 b^{2} \lambda N+6 b^{2} \lambda-2 b m \lambda-5 b m \lambda^{2}+4 \lambda^{2}+3 b m-4 \lambda\right) \eta^{5}\right) \\
& +\mathrm{O}\left(\eta^{6}\right)
\end{aligned}
$$

and $F(\eta)=\lim _{n \rightarrow \infty} F_{n}$.

\section{Padé approximation technique}

It is now well known that Padé approximants [17] have the advantage of manipulating the polynomial approximation into rational functions of polynomials. By this manipulation we gain more information about the mathematical behavior of the solution. In addition, power series is not useful for large values of $\eta$. Boyd [18] and others have formally shown that power series in isolation are not useful to handle boundary value problems. This can be attributed to the possibility that the radius of convergence may not be sufficiently large to contain the boundaries of the domain. It is therefore essential to combine the series solution, obtained by the ADM and VIM or any series solution methods, with the Padé approximants to provide 
Table 1: Comparison of the values of $F^{\prime \prime}(0)$ with previous results for $N=0, \lambda=1$.

\begin{tabular}{ccccc}
\hline$m$ & Postelnicu et al. [6] & Ali [5] & Present results (Padé [3/3]) & Present results (Padé [6/6]) \\
\hline-1.0 & -0.6180 & -0.61803 & -0.61728 & -0.61803 \\
-0.8 & -0.6770 & -0.67703 & -0.67516 & -0.67703 \\
-0.6 & -0.7440 & - & -0.74412 & -0.74404 \\
-0.4 & -0.8198 & -0.81980 & -0.82101 & -0.81982 \\
-0.2 & -0.9050 & - & -0.90513 & -0.90499 \\
0.0 & -1.0000 & -1.00000 & -0.99998 & -1.00000 \\
0.2 & -1.1049 & - & -1.10524 & -1.10497 \\
0.4 & -1.2198 & - & -1.22781 & -1.21976 \\
0.6 & -1.3440 & - & -1.34462 & -1.34390 \\
0.8 & -1.4770 & - & -1.47789 & -1.47701 \\
1.0 & -1.6180 & -1.61803 & -1.62351 & -1.61803 \\
\hline
\end{tabular}

Table 2: Numerical values of $F^{\prime \prime}(0)$ for $N>0$ using Padé approximants [6/6].

\begin{tabular}{lccc}
\hline$N$ & $\lambda$ & $m$ & $F^{\prime \prime}(0)$ \\
\hline 1.0 & 1.0 & 1.0 & -0.999822 \\
2.0 & 1.0 & 1.0 & -0.767530 \\
3.0 & 1.0 & 1.0 & -0.640354 \\
4.0 & 1.0 & 1.0 & -0.593069 \\
5.0 & 1.0 & 1.0 & -0.528624 \\
1.0 & 1.0 & 0.0 & -0.707100 \\
1.0 & 1.0 & 0.5 & -0.843048 \\
1.0 & 1.0 & -0.5 & -0.593084 \\
1.0 & 1.0 & -1.0 & -0.500004 \\
\hline
\end{tabular}

an effective tool to handle boundary value problems on an infinite or semi-infinite domain. Recall that the Padé approximants can be easily evaluated by using built-in function in a symbolic computational package such as Maple. The essential behavior of the solution will be addressed by using several diagonal Padé approximants of different degrees. Furthermore, the undetermined value of $b=F^{\prime \prime}(0)$ is calculated from the boundary condition at infinity in (2.14). The difficulty at infinity is overcome by employing the diagonal Padé approximants $[10,11,18]$ that approximate $F^{\prime}(\eta)$ using $W_{L}^{\prime}(\eta)$. For instance, the series is transformed into diagonal Padé approximants as follows:

$$
W_{L[M, M]}^{\prime}(\eta)=\frac{\sum_{i=0}^{M} h_{i} \eta^{i}}{\sum_{i=0}^{M} g_{i} \eta^{i}}
$$

where $P=2(M+1)$ is the order of the series required for each approximant. In the Maple environment, the simultaneous evaluation of $\lim _{\eta \rightarrow \infty} W_{L[M / M]}^{\prime}(\eta)=0$ for $M=2,3,4, \ldots$ in (3.9) gives the numerical results for $b=F^{\prime \prime}(0)$ as shown in Tables 1 and 2. 


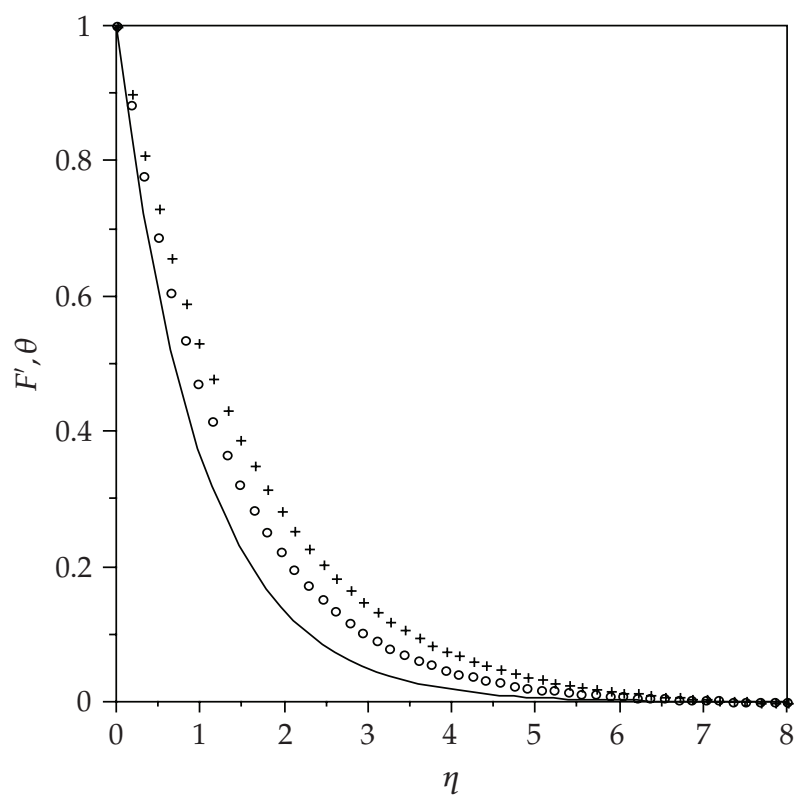

Figure 1: Vertical velocity or temperature profiles for $\lambda=1 ; m=1 ; N=1$ (solid line), $N=2$ (circles), $N=3$ (plus signs).

\section{Numerical results and discussion}

The governing equation (2.13) subject to the boundary conditions (2.14) is solved using both $\mathrm{ADM}$ and VIM together with Padé technique as described in Sections 3-5. Solutions are obtained for the plate temperature with uniform lateral mass flux $(\lambda=1)$ controlled by the suction/injection parameter $m$ and radiation parameter $N$ as shown in Tables 1 and 2 and Figures 1 and 2.

The results presented in Table 1 are in good agreement with those given by Postelnicu et al. [6] and Ali [5] who solved numerically the case of permeable surface without considering the thermal radiation effect. In Table 2, we observed that the local surface heat flux rate decreases with increasing values of radiation parameter. Figures 1 and 2 confirm the exponential decay velocity $F^{\prime}(\eta)$ or temperature $\theta(\eta)$ profiles across the boundary layers [5-8]. As mentioned earlier, suction corresponds to $m>0$, injection to $m<0$, and $m=0$ to impermeable plate. Therefore, it is clear from Figure 1 that suction reduces the boundary layer thickness sharply as seen for $m=1$ while injection increases it as for $m=-1$; however, the surface heat flow is always positive regardless of the sign of $m$ where the heat is directed from the plate to the porous medium. Figure 2 shows that the fluid velocity and temperature increase as the radiation parameter $N$ increases. This can be explained by the fact that the effect of radiation $N$ is to increase the rate of energy transport to the fluid and accordingly to increase the fluid temperature.

\section{Conclusions}

We employed both ADM and VIM to compute a nonperturbative solution for thermal radiation effect on natural convection boundary layer flow past a vertical plate embedded in a saturated porous medium. The results demonstrate the reliability and the efficiency 


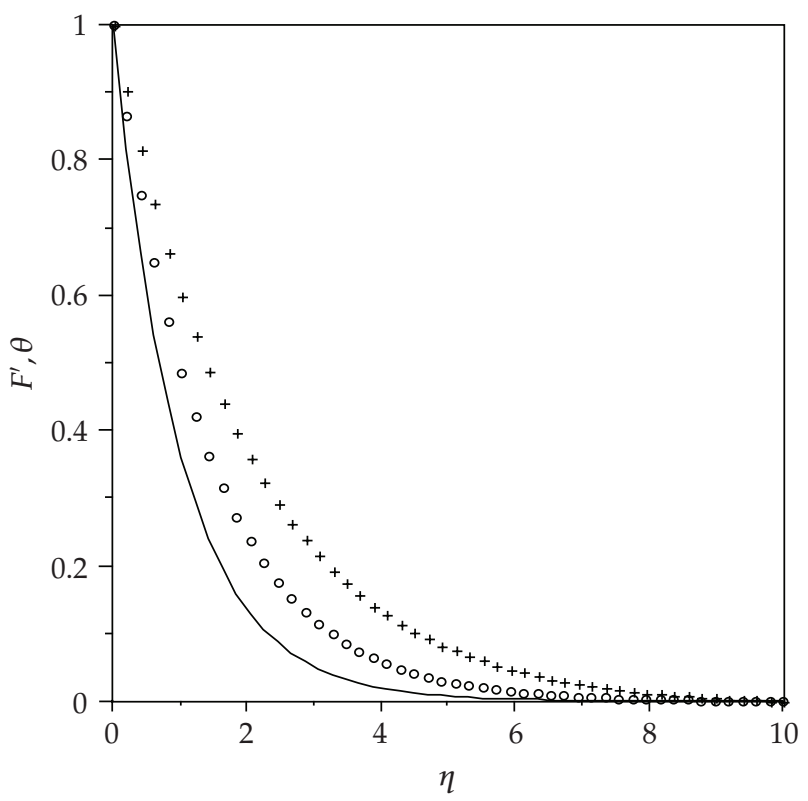

Figure 2: Vertical velocity or temperature profiles for $N=1 ; \lambda=1 ; m=1$ (solid line), $m=0$ (circles), $m=-1$ (plus signs).

of both methods in an unbounded domain. The two methods are powerful and efficient in obtaining approximations of higher accuracy and closed-form solutions if existing. However, He's variational iteration method gives several successive approximations through using the iteration of the correction functional and Adomian decomposition method provides the components of the exact solution that will be added to get the series solution. Moreover, the VIM requires the evaluation of the Lagrangian multiplier whereas ADM requires the evaluation of the Adomian polynomials that mostly require tedious algebraic calculations.

\section{Acknowledgment}

The authors would like to thank the National Research Foundation of South Africa Thuthuka programme for financial support.

\section{References}

[1] D. A. Nield and A. Bejan, Convection in Porous Media, chapter 5, Springer, New York, NY, USA, 2nd edition, 1999.

[2] A. Nakayama and H. Koyama, "Free convective heat transfer over a non-isothermal body of arbitrary shape embedded in a fluid saturated porous medium," Journal of Heat Transfer, vol. 109, no. 1, pp. 125130, 1987.

[3] P. Cheng and W. J. Minkowycz, "Free convection about a vertical flat plate embedded in a porous medium with application to heat transfer from a dike," Journal of Geophysical Research, vol. 82, no. 14, pp. 2040-2044, 1977.

[4] P. Cheng, "The influence of lateral mass flux on free convection boundary layers in a saturated porous medium," International Journal of Heat and Mass Transfer, vol. 20, no. 3, pp. 201-206, 1977.

[5] M. E. Ali, "The effect of lateral mass flux on the natural convection boundary layers induced by a heated vertical plate embedded in a saturated porous medium with internal heat generation," International Journal of Thermal Sciences, vol. 46, no. 2, pp. 157-163, 2007. 
[6] A. Postelnicu, T. Groşan, and I. Pop, "Free convection boundary-layer over a vertical permeable flat plate in a porous medium with internal heat generation," International Communications in Heat and Mass Transfer, vol. 27, no. 5, pp. 729-738, 2000.

[7] O. D. Makinde, "Free convection flow with thermal radiation and mass transfer past a moving vertical porous plate," International Communications in Heat and Mass Transfer, vol. 32, no. 10, pp. 1411-1419, 2005.

[8] T. Groşan and I. Pop, "Free convection of non-Newtonian fluids over a vertical surface in a porous medium with internal heat generation," International Journal of Applied Mechanics and Engineering, vol. 7, no. 2, pp. 401-407, 2002.

[9] G. Adomian, Solving Frontier Problems of Physics: The Decomposition Method, vol. 60 of Fundamental Theories of Physics, Kluwer Academic Publishers, Dordrecht, The Netherlands, 1994.

[10] A.-M. Wazwaz, "The modified decomposition method and Padé approximants for a boundary layer equation in unbounded domain," Applied Mathematics and Computation, vol. 177, no. 2, pp. 737-744, 2006.

[11] S. A. Kechil and I. Hashim, "Non-perturbative solution of free-convective boundary-layer equation by Adomian decomposition method," Physics Letters A, vol. 363, no. 1-2, pp. 110-114, 2007.

[12] T. A. Abassy, M. A. El-Tawil, and H. K. Saleh, "The solution of Burgers' and good Boussinesq equations using ADM-Padé technique," Chaos, Solitons Eamp; Fractals, vol. 32, no. 3, pp. 1008-1026, 2007.

[13] J.-H. He and X.-H. Wu, "Variational iteration method: new development and applications," Computers Eamp; Mathematics with Applications, vol. 54, no. 7-8, pp. 881-894, 2007.

[14] J.-H. He, "Variational iteration method-some recent results and new interpretations," Journal of Computational and Applied Mathematics, vol. 207, no. 1, pp. 3-17, 2007.

[15] J.-H. He and X.-H. Wu, "Construction of solitary solution and compacton-like solution by variational iteration method," Chaos, Solitons Eamp; Fractals, vol. 29, no. 1, pp. 108-113, 2006.

[16] J.-H. He, "Some asymptotic methods for strongly nonlinear equations," International Journal of Modern Physics B, vol. 20, no. 10, pp. 1141-1199, 2006.

[17] G. A. Baker Jr., Essentials of Padé Approximants, Academic Press, London, UK, 1975.

[18] J. Boyd, "Padé approximant algorithm for solving nonlinear ordinary differential equation boundary value problems on an unbounded domain," Computers in Physics, vol. 11, no. 3, pp. 299-303, 1997.

[19] M. A. Seddeek, "Thermal radiation and buoyancy effects on MHD free convection heat generation flow over an accelerating permeable surface with temperature dependent viscosity," Canadian Journal of Physics, vol. 79, no. 4, pp. 725-732, 2001.

[20] M. Inokuti, H. Sekine, and T. Mura, "General use of the Lagrange multiplier in nonlinear mathematical physics," in Variational Method in the Mechanics of Solids, S. Nemat-Nassed, Ed., pp. 156-162, Pergamon press, New York, NY, USA, 1978. 




Advances in

Operations Research

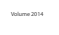



\section{The Scientific} World Journal
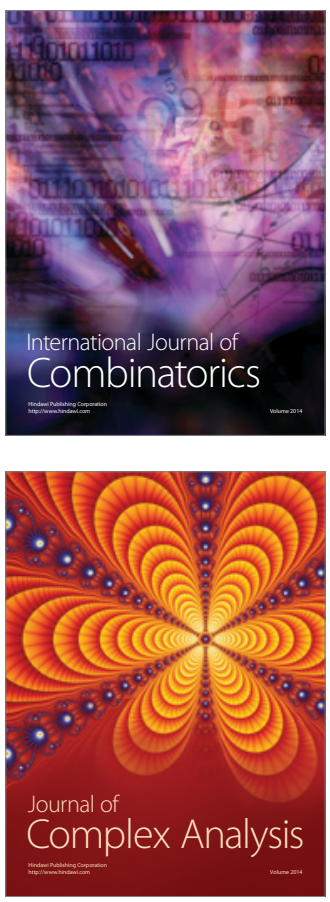

International Journal of

Mathematics and

Mathematical

Sciences
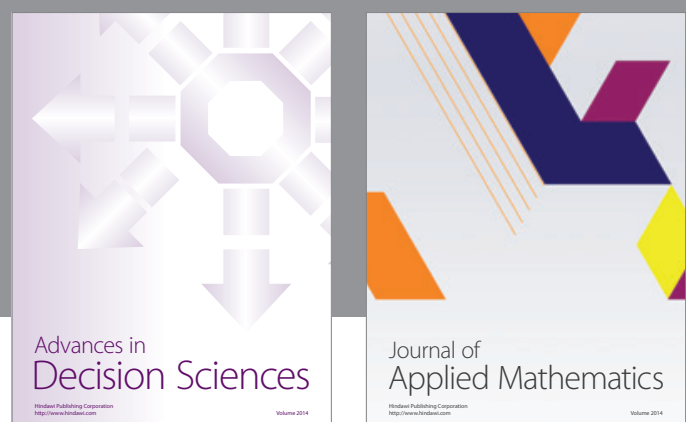

Journal of

Applied Mathematics
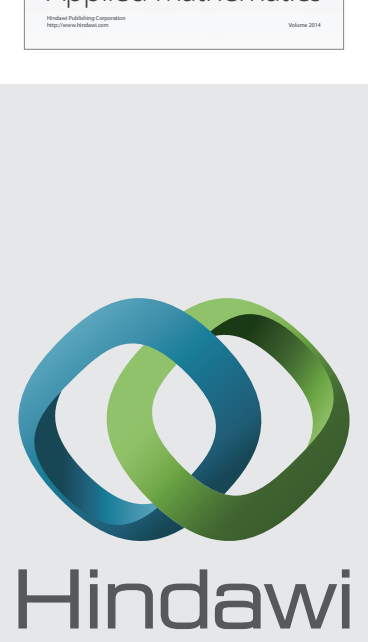

Submit your manuscripts at http://www.hindawi.com
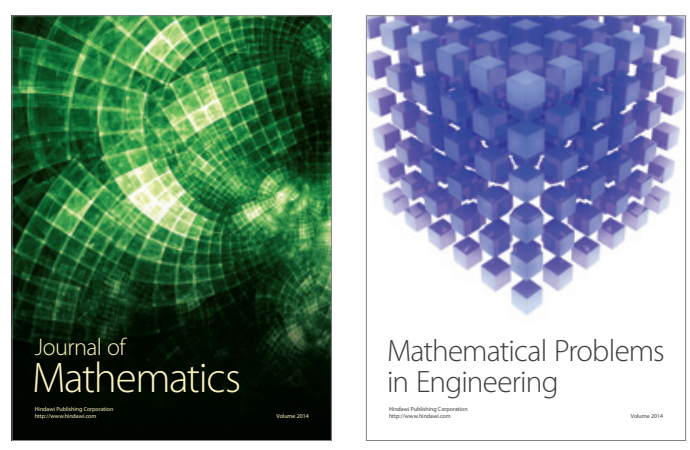

Mathematical Problems in Engineering
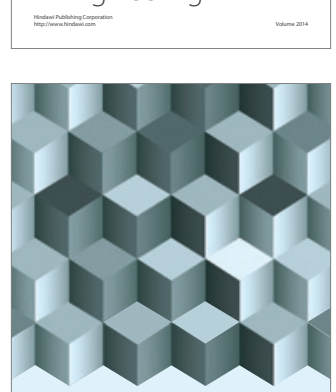

Journal of

Function Spaces
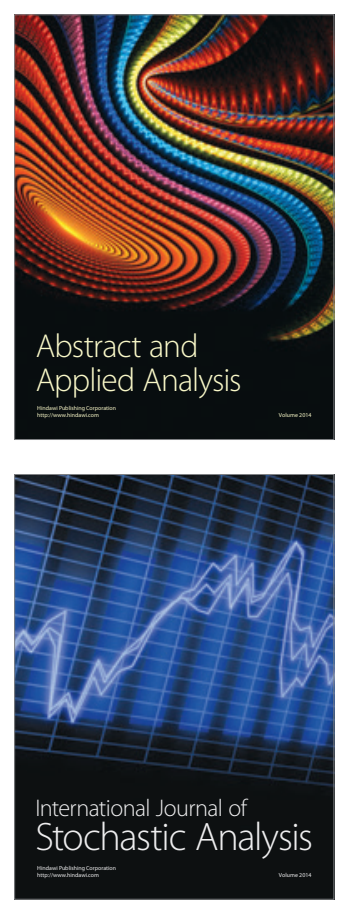



ournal of

Probability and Statistics

Promensencen
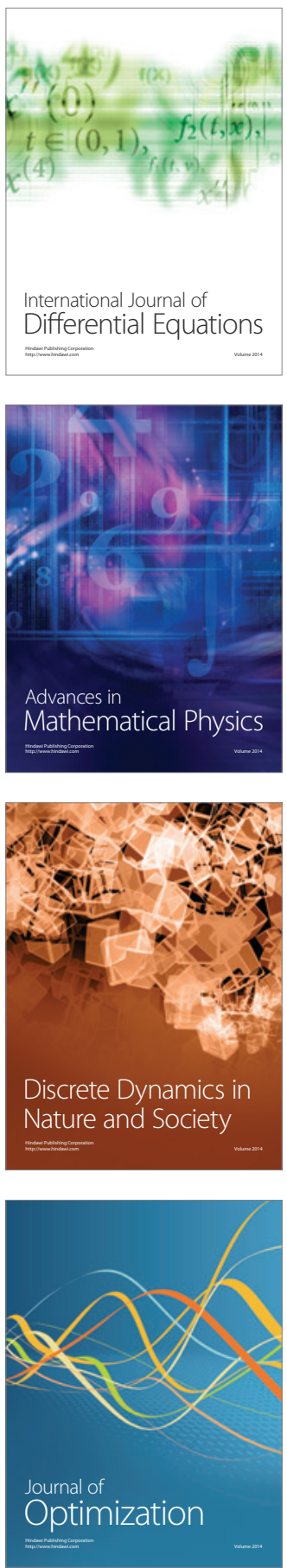